\title{
Disomie uniparentale du chromosome 16 et mosaique placentaire
}

Dans un article paru en novembre 1992, André Boué insistait [1] sur la fréquence des anomalies chromosomiques au début de la gestation. Il estimait que sur 1000 grossesses ayant atteint 10 semaines, la coexistence d'une trisomic, complète ou en mosaïque, du placenta et d'un embryon diploïde, sc rencontre en moyenne six fois. Au cours du développement du foctus, un des trois chromosomes disparaît et, dans un tiers des cas, c'est celui du parent qui n'en avait fourni qu'un. Une disomie uniparentale se produirait ainsi dans deux grossesses sur 1000 , ce qui, pour 750000 naissances, ferait un total d'environ 1500 nouveau-nés porteurs d'une disomie uniparentale, c'est-à-dire d'un chromosome dont les deux exemplaires proviendraient d'un seul parent. On ne s'aperçoit de ce phénomène que lorsqu'une anomalie conduit à le rechercher, et cela dans deux cas principaux : quand les deux chromosomes sont identiques (isodisomic), une anomalic récessive peut se révéler, alors qu'un scul des parents est porteur du trait; ou quand une "empreinte parentale " a comme résultat une anomalie si les deux chromosomes viennent d'un scul parent, la contribution des deux sexes étant indispensable ; l'exemple le mcilleur reste le syndrome de Prader-Willi, dont $\mathrm{m} / \mathrm{s}$ a parlé à plusicurs reprises $\left(n^{\circ} 1\right.$, vol. 6, p. 57 et $n^{\circ} 7$, vol. 8, p. 741).

Une étude systématique devrait donc révéler un nombre relativement élevé de disomies uniparentales lorsque l'examen des villosités choriales a montré une trisomie complète ou partielle, non confirmé par celui d'une ponction amniotique et le caryotype à la naissance. Cette recherche pourrait également évaluer les risques que crée cette situation. Une telle étude, la première à notre connaissance, a été entreprise en collaboration entre des équipes canadienne, américaine et argentine [2] $\mathrm{m} / \mathrm{s} n^{\circ} 4$ vol. 9, avril 93 et porte sur le chromosome 16. Un travail préliminaire avait été présenté à un congrès comme le rapport par $\mathrm{A}$. Boué [1]

L'échantillon était formé de neuf cas chez lesquels un diagnostic prénatal de mosaïcisme placentaire avait été posé, l'indication du prélèvement chorionique étant, soit l'âge de la mère, soit un retard de croissance in utero.

Sur le plan biologique, le fait essentiel était que, dans tous les cas, la biopsie chorionique, directe ou après culture, montrait $100 \%$ de trisomic 16 ; au contraire, l'amniocentèse effectuéc au $2^{e}$ trimestre et le foctus (sang de cordon) indiquait $0 \%$. En revanche, des différences existaicnt dans le placenta à terme, dont la plupart conservait un pourcentage élevé de cellules trisomiques. L'analyse de l'origine des cellules montra deux faits importants : dans les villosités choriales, tous les cas interprétables (7 sur 9) avaient un chromosome supplémentaire d'origine maternelle ; en outre, sur huit enfants interprétables, quatre avaient hérité leurs chromosomes 16 de leurs deux parents, et quatre ne portaient que des chromosomes maternels; trois au moins étaient hétérodisomiques, c'està-dire que les deux chromosomes maternels étaient présents. Parmi les enfants porteurs de disomie maternelle, deux ne sont pas allés à terme : l'un est mort spontanément à 20 semaines, sans présenter de lésions apparentes; le deuxième a été victime d'une interruption pour hypertension maternelle ; un troisième avait une imperforation anale isolée opérée avec succès. Ces résultats sont trop hétérogènes pour qu'on puisse incriminer la disomie uniparentale dans les troubles observés, plutôt que, par exemple, le niveau de mosaïcisme placentaire à la naissance. Il n'est donc pas possible actuellement de conclure à la nocivité d'une disomie maternelle du 16 , et une observation prolongée des enfants survivants sera nécessaire. Il est souhaitable que de telles observations soient étendues aux autres chromosomes sujets à de telles évolutions, dont les plus communs, outre le 16 et le 21 , sont le 7 , le 8 , le 15 et le 18 . Des résultats ont déjà été obtenus pour le $15: \mathrm{m} / \mathrm{s}$ a rapporté un exemple de syndrome de Prader-Willi après "disomisation" d'une mosaïque trisomique $\left(n^{\circ} 7\right.$, vol. 8, p. 741); pour le 7 , la suspicion qu'une disomie maternelle entraîne une diminution de la taille pourrait être confirmée ou infirmée par une étude de ce type $\left(n^{\circ} 3\right.$, vol. 9, p. 333).

Une dernière remarque : dans les disomies uniparentales, la prédominance des contributions maternelles a fait soulever diverses hypothèses, dont celle de la létalité d'une contribution éventuelle uniquement paternelle. Il semble qu'elle résulte plus simplement de la fréquence beaucoup plus grande de l'origine maternelle du chromosome surnuméraire, comme cela est bien démontré dans le cas de la trisomie 21 $\left(\mathrm{m} / \mathrm{s} n^{\circ} 5\right.$, vol. 7, p. 520).

J.C.D.

1. Boué A. L'instabilité chromosomique des premières mitoses blastomériques. médecine/sciences 1992 ; 8 : 929-37.

2. Kalousek DK, Langlois S, Barrett I, Yam I, Wilson DR, Howard-Peebles PN, Johnson MP, Giorgiutti E. Uniparental disomy for chromosome 16 in humans. Am J Hum Gond 1993 ; 52 : 8-16. 